\title{
proBDNF Is a Major Product of $b d n f$ Gene Expressed in the Perinatal Rat Cortex
}

\author{
P. N. MENSHANOV ${ }^{1,2^{*}}$, D. A. LANSHAKOV ${ }^{1^{*}}$, N. N. DYGALO ${ }^{1,2}$ \\ " These authors contributed equally to this work.
}

${ }^{1}$ Functional Neurogenomics Laboratory, Institute of Cytology and Genetics Siberian Branch of Russian Academy of Sciences, Novosibirsk, Russian Federation, ${ }^{2}$ Department of Natural Sciences, Novosibirsk State University, Novosibirsk, Russian Federation

Received January 14, 2015

Accepted April 17, 2015

On-line June 5, 2015

\section{Summary}

In the developing brain, mature brain derived neurotrophic factor (mBDNF) and its precursor (proBDNF) exhibit prosurvival and proapoptotic functions, respectively. However, it is still unknown whether mBDNF or proBDNF is a major form of neurotrophin expressed in the immature brain, as well as if the level of active caspase- 3 correlates with the levels of BDNF forms during normal brain development. Here we found that both proBDNF and mBDNF were expressed abundantly in the rat brainstem, hippocampus and cerebellum between embryonic day 20 and postnatal day 8 . The levels of mature neurotrophin as well as mBDNF to proBDNF ratios negatively correlated with the expression of active caspase- 3 across brain regions. The immature cortex was the only structure, in which proBDNF was the major product of $b d n f$ gene, especially in the cortical layers 2-3. And only in the cortex, the expression of BDNF precursor positively correlated with the levels of active caspase-3. These findings suggest that proBDNF alone may play an important role in the regulation of naturally occurring cell death during cortical development.

\section{Key words}

Developing brain $\bullet$ Cerebral cortex $\bullet$ proBDNF $\bullet$ mBDNF $\bullet$ Active caspase-3

\section{Corresponding author}

P. N. Menshanov, Functional Neurogenomics Laboratory, Institute of Cytology and Genetics, Russian Academy of Science, Lavrentyev av. 10, Novosibirsk, 630090, Russian Federation. Fax: +7-383-333-12-78. E-mail: MenshanovPN@icg.sbras.ru

\section{Introduction}

Mature brain derived neurotrophic factor (mBDNF) is an important regulator of cell survival and plasticity in the immature brain (Ghosh et al. 1994, Dekkers et al. 2013). It is thought that the cerebral levels of mBDNF can affect the levels of key apoptotic executor enzyme - active caspase-3 under pathological and trauma conditions (Han et al. 2000). Thus, for example, the pretreatment with mBDNF prevents caspase-3 activation induced by hypoxic-ischemia or axotomy in the developing cortex (Han et al. 2000, Han and Holtzman 2000, Giehl et al. 2001, Hung et al. 2013, Nakajima et al. 2010). The increased levels of endogenous mBDNF also provide the resistance to ischemic brain damage (Kokaia et al. 1996).

However, in vivo absence of mBDNF expression during normal development of cortex resulted in the enhanced cell death of subventricular zone neurons only, while other cell populations in this brain region remained unaffected (Linnarsson et al. 2000). Moreover, the death of transplanted cortical interneurons was not affected by the cell-autonomous disruption of $\mathrm{TrkB}$, the main neurotrophin receptor expressed by neurons in the CNS (Southwell et al. 2012), making the role of mBDNF in the normal brain development less clear.

Recent studies have shown that BDNF precursor - proBDNF is able to avoid conversion by furin, plasmin or prohormone convertases, can be secreted from the cell like its molecular descendant - mBDNF, and exerts biological proapoptotic activity as a specific ligand for 
p75NTR receptor (Teng et al. 2005, Fayard et al. 2005, Kenchappa et al. 2006, Wang et al. 2006, Fan et al. 2008, Lessmann and Brigadski 2009, Yang et al. 2009, Xu et al. 2011). Several reports demonstrated the presence of the proneurotrophin in the immature CNS (Tang et al. 2008, 2010, Muzyka et al. 2012), but the ratio of mBDNF to proBDNF was not yet defined during perinatal brain development (Perovic et al. 2013).

Both forms of BDNF exert their apoptotic effects via a caspase-3-dependent molecular pathway, and the predominance of one form over the other may influence the caspase-3 activation in the immature nervous system (Han et al. 2000, Madeddu et al. 2004, Koshimizu et al. 2010). Proapoptotic action of proBDNF could be antagonized by mBDNF (Koshimizu et al. 2010). ProBDNF is also suspected to play a functional role in anesthesia-induced apoptosis of neurons (Head et al. 2009). However, it is still unknown if the levels of mBDNF or proBDNF correlate in vivo with the expression of active caspase- 3 in the brain regions that differ in the age-related pattern of developmental naturally occurring cell death, like cortex and brainstem (Mooney and Miller 2000, Menshanov et al. 2006).

Therefore, the aim of the study is to determine the levels of BDNF forms - proapoptotic proneurotrophin and antiapoptotic mature neurotrophin in the immature brain during perinatal development, and to find out if the expression of the key apoptotic marker - active caspase-3 is correlated with the levels of these proteins across brain regions.

\section{Materials and Methods}

\section{Materials}

For western blotting, polyclonal primary rabbit antibodies (N-20 antibody for mBDNF and proBDNF; H-277 for procaspase-3 and active caspase-3; I-19-R for actin) and secondary goat anti-rabbit alkaline phosphatase conjugated antibodies were obtained (Santa Cruz, USA). For immunohistochemistry, polyclonal primary chicken ab78367 antibody for proBDNF (Abcam, UK), polyclonal rabbit H-117 antibody for mBDNF (Santa Cruz, USA) and monoclonal rabbit 5A1E antibody for active caspase-3 (Cell signaling, USA), as well as secondary donkey anti-chicken DyLight 594 and donkey anti-rabbit Dylight 488 antibodies (Jackson ImmunoResearch Europe Ltd) were purchased. TissueTek optimal cutting temperature (OCT) embedding matrix was obtained from Sakura Finetek Europe B.V.
(Leiden, Netherlands). All other chemicals and reagents were of analytical grade and were purchased from Sigma Chemical Company (St. Louis, USA) or Bio-Rad Laboratories (Hercules, USA).

\section{Animals}

Wistar rats bred in the animal facilities at the Institute of Cytology and Genetics, Novosibirsk, were used. The colony was maintained under natural illumination at $22-24{ }^{\circ} \mathrm{C}$ with food and water available ad libitum. All animal use procedures were in compliance with the European Directive 2010/63/EU. The study was approved by the Bioethical Committee of the Institute of Cytology and Genetics Siberian Branch of Russian Academy of Sciences, Novosibirsk. All efforts were made to minimize animal suffering and to use only the number of animals necessary to produce reliable data.

Adult female rats weighing 200-250 g were mated with males in late afternoon. Vaginal smears were taken in the morning. The day of conception was defined as embryonic day 1 , and the day of birth - as postnatal day 1. Litters were culled after birth to 8 pups of both sexes on the postnatal day 2 . No more than two pups from any one litter were used for experiment.

For immunoblot, tissue samples of frontal cortex and brainstem were taken from 20-day-old fetuses (E20; $\mathrm{n}=7$ ), 4 and 8-day-old pups ( $\mathrm{P} 4 ; \mathrm{n}=7$ and $\mathrm{P} 8 ; \mathrm{n}=7$ ) and 115 -day-old adult rats $(\mathrm{P} 115 ; \mathrm{n}=7)$. For that purpose, animals were rapidly decapitated and samples were dissected out on a cooled plate and frozen in liquid nitrogen until further processing. Frontal cortex sample included $1.5 \mathrm{~mm}$ thick cut from the upper surface of the frontal half of the hemispheres. Brainstem sample included pons and medulla oblongata. Hippocampi and cerebella were taken from the same P4 animals for interregional comparison. In addition, another five P4 animals were used for immunohistochemistry.

\section{Immunoblot analysis}

Brain tissue was homogenized in lysis buffer containing $150 \mathrm{mM} \mathrm{NaCl}, 50 \mathrm{mM}$ Tris, $1 \%$ Triton X-100 and following protease inhibitors: $2 \mathrm{mM}$ phenylmethylsulfonylfluoride and $2 \mu \mathrm{g} / \mathrm{ml}$ of leupeptin, pepstatin and aprotinin. Electrophoresis was used to separate aliquots $(50 \mu \mathrm{g})$ of total protein on $16.5 \%$ sodium dodecyl sulfate polyacrylamide gel in Mini-Protean 3 Dodeca Cell (Bio-Rad Laboratories, USA). The resolved proteins were transferred on to nitrocellulose membrane by Transblot Cell (Bio-Rad Laboratories, USA). Ponceau S staining 
was used to control equal loading of the samples and protein transfer to membrane. Detection of apoptotic proteins was performed as it was described previously (Menshanov et al. 2006, Men'shanov et al. 2011) with polyclonal primary rabbit antibodies (dilution 1:200 for mBDNF and proBDNF; 1:200 for procaspase-3 and active caspase-3; 1:1000 for actin) and secondary alkaline phosphatase conjugated goat anti-rabbit antibody (1:500). ProBDNF was detected as a single band at $32 \mathrm{kDa}$, $\mathrm{mBDNF}$ at $14 \mathrm{kDa}$ and procaspase- 3 at $32 \mathrm{kDa}$. Active caspase- 3 bands were detected at 20,17 and $12 \mathrm{kDa}$. Actin with a single band at $42 \mathrm{kD}$ was used as an internal loading control. Intensities of the signals for mBDNF, proBDNF, procaspase-3 and active caspase- 3 bands were in a range of a linear dependence on these proteins levels. Specificity of mBDNF and proBDNF detection with N20 antibodies was confirmed by their absence in BDNF knockout brain lysates previously in several reports (for example Nagappan et al. 2009, Egashihara et al. 2010).

\section{Immunohistochemistry, microscopy, and image analysis}

At P4 pups were anesthetized with avertin and transcardially perfused with $1 \mathrm{x}$ PBS followed $4 \%$ PFA in PBS. Brains were postfixed with $4 \%$ paraformaldehyde, cryoprotected with $30 \%$ sucrose overnight, and embedded in Tissue-Tek OCT embedding matrix. A total of $15 \mu \mathrm{m}$ coronal sections were processed for immunohistochemistry as it was described previously (Lanshakov et al. 2009). Briefly, slides were incubated with primary chicken antibody for proBDNF (1:100) and primary rabbit antibody for mBDNF (1:100) / primary rabbit antibody for active caspase-3 (1:200), followed by incubation with a donkey anti-chicken DyLight 594-conjugated antibody (1:500) and secondary donkey anti-rabbit DyLight 488-conjugated antibody (1:500), respectively. All cryosections were counterstained with DAPI. Tissue sections were viewed on a Zeiss LSM 780 microscope (Carl Zeiss Microscopy $\mathrm{GmbH}$, Oberkochen, Germany), and images were collected and analyzed with ZEN Software (Carl Zeiss Microscopy $\mathrm{GmbH}$, Jena, Germany). Mean optical density was calculated in ZEN, as a rectangular profile through frontal cortex. Dimensions of rectangular zone were equal for all measurements. Images were optimized for size, color, and contrast using Photoshop CS4 (Adobe Systems Inc., San Jose, USA).

\section{Data analysis}

All data are represented as means \pm SEM. Data on protein levels were analyzed by 1-way (simple factor -
Region), 2-way (simple factors - Age and Region) or 2-way mix-design ANOVA (simple factor-BDNF form, repeated measures factor - Distribution). Statistical comparison of between group differences was performed using Fisher LSD post-hoc test. The relationship between protein levels was estimated by the Spearman rank correlation test followed by the linear regression analysis. The results were considered significant at probability level less than 0.05. All data analysis was performed using the Statistica 12.

\section{Results}

$m B D N F$ and proBDNF levels in the cortex and brainstem The levels of mBDNF and proBDNF were analyzed in the cortex and brainstem of E20, P4 and P8 animals by immunoblot, and were compared to the levels of these proteins in the adult brain. The expression of proBDNF in the cerebral cortex did not change significantly between E20 and P8 days and was two to three times lower in comparison with the proneurotrophin expression in the adult brain (Fig. 1a,c; Age $\left.\mathrm{F}_{(3,20)}=33.18, \mathrm{p}<0.0001\right)$. Unlike proBDNF, mBDNF was almost undetectable in all samples of the cortices of E20, P4 and P8 animals (Fig. 1a,b; BDNF form $\left.\mathrm{F}_{(1,15)}=210.98, \quad \mathrm{p}<0.0001\right)$. Mean cortical ratio of mBDNF to proBDNF did not exceed 0.05-0.10 during perinatal development (Fig. 1d; Age $-\mathrm{F}_{(3,18)}=11.42$, $\mathrm{p}<0.0002)$. In contrast with the developing cortex, mature neurotrophin was readily detectable in all cortical samples obtained from adult rats (Fig. 1a,b; Age $\left.\mathrm{F}_{(3,19)}=158.57, \mathrm{p}<0.0001\right)$, and $\mathrm{mBDNF}$ to proBDNF ratio was five to ten times higher at this age in comparison with this ratio in the perinatal samples (Fig. 1d; Age $-\mathrm{F}_{(3,18)}=11.42, \mathrm{p}<0.0002$ ).

The levels of proBDNF in the developing brainstem did not vary significantly on E20, P4 and P8 days (Fig. 1a,c; Age $-\mathrm{F}_{(3,21)}=18.61, \mathrm{p}<0.0001$ ), and were two times higher than levels of proneurotrophin in the immature cortex (Fig. 1c; Region $-\mathrm{F}_{(1,34)}=95.45$, $\mathrm{p}<0.0001)$. However, the expression of proBDNF in the brainstem samples was about two times lower than the expression of the mature neurotrophin (Fig. 1a-c; BDNF form $\left.-F_{(1,10)}=18.91, p<0.0015\right)$. This resulted in the high, equal to $1.5-2.0, \mathrm{mBDNF}$ to proBDNF ratio in this brain region during perinatal development (Fig. 1d; Age $\left.\mathrm{F}_{(3,16)}=8.64, \mathrm{p}<0.0013\right)$. The mBDNF to proBDNF ratio in the developing brainstem was three to five times higher than in the adult brainstem and cortex, in which it was 
about $0.4-0.5$, and more than one order of magnitude higher than in the developing cortex (Fig. 1d).

These data suggested that, in contrast to adult brain, the immature cortex had the low $\mathrm{mBDNF} /$ proBDNF ratio, which was constant through all perinatal development.
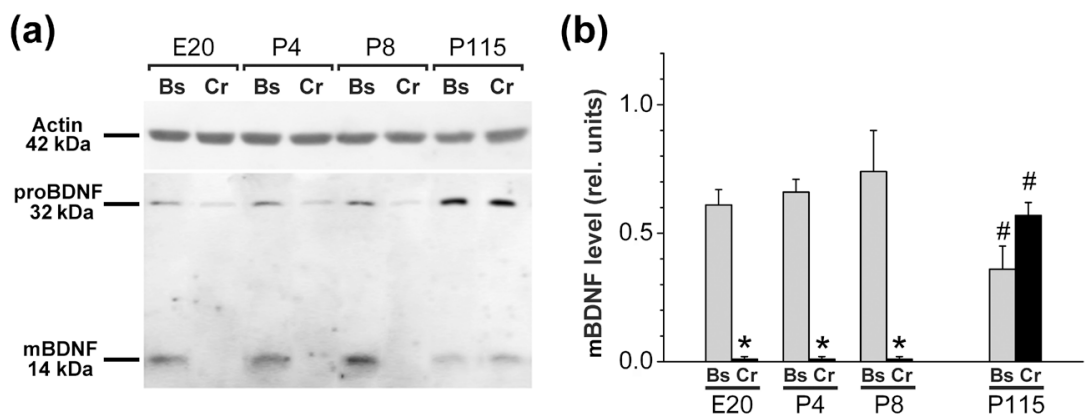

(c)

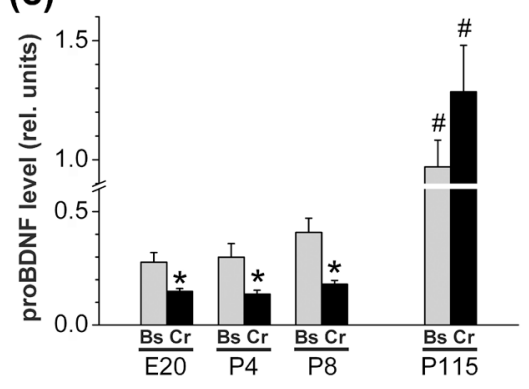

(d)

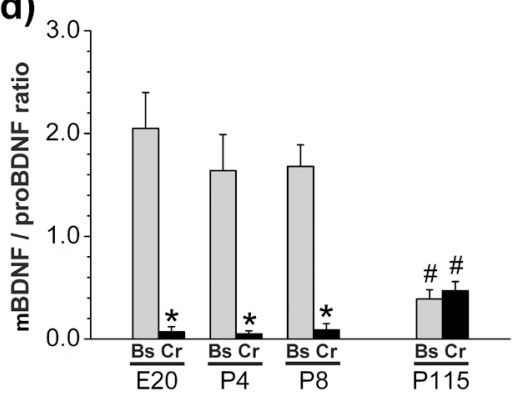

(a)
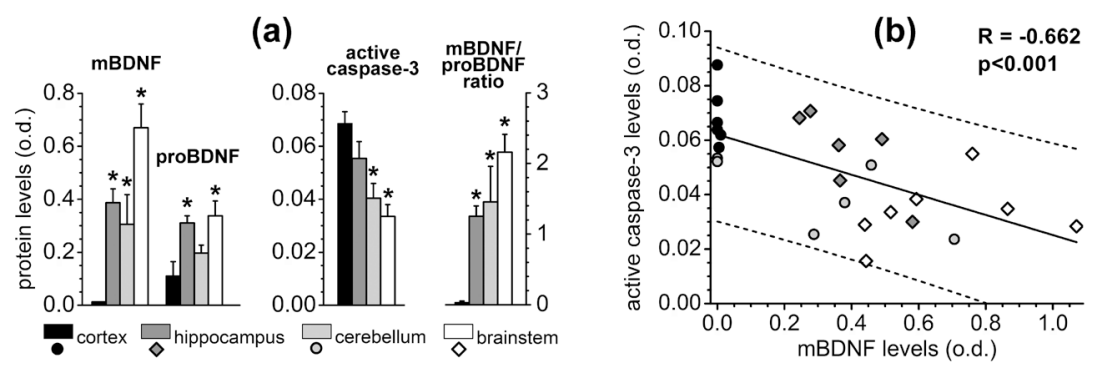

(c)

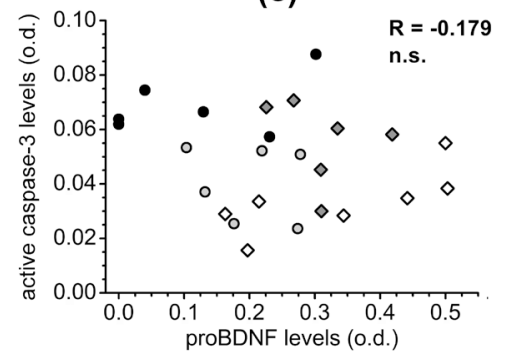

Fig. 1. Expression of mBDNF and proBDNF in the rat cortex and brainstem. (a) Immunoblot with mBDNF (14 kDa), proBDNF (32 kDa) and actin (42 kDa) bands. (b) mBDNF protein levels. (c) proBDNF protein levels. (d) mBDNF to proBDNF ratio. * $-p<0.05$ vs brainstem. \# $-\mathrm{p}<0.05$ vs previous age. E20 - 20-dayold fetuses, P4 - 4-day-old pups, P8 8-day-old pups, P115 - 115-day-old adult rats. Bs - brainstem. Cr - cortex. Data are presented as the means \pm SEM.
Fig. 2. $m B D N F$, proBDNF and active caspase-3 expression in the brain regions of P4 rats. (a) mBDNF, proBDNF, active caspase-3 protein levels and mBDNF/ proBDNF ratio in the cortex, hippocampus, cerebellum and brainstem on P4. * $-p<0.05$ vs level in the cortex. Data are presented as the means \pm SEM. (b) Association between mBDNF and active caspase-3 levels across brain regions. (c) Lack of the association between levels of proBDNF and active caspase-3. (d) Association of $\mathrm{mBDNF} / \mathrm{proBDNF}$ ratio and the level of active caspase- 3 in the brain regions. Solid lines mark the regressions; short dot lines mark $95 \%$ prediction intervals for regressions. $m B D N F$, proBDNF and active caspase-3 levels in the neonatal brain regions

To find out if the prevalence of proBDNF over mBDNF was unique to immature cortex, the cortical levels of BDNF forms were compared to the levels of these proteins in the brain structures that differ in the expression of active caspase-3 during development (Menshanov et al. 2006). The amount of active caspase-3 on P4 was highest in the cortex and lowest in the cerebellum and brainstem (Fig. 2a; $\mathrm{F}_{(3,21)}=9.18$, $\mathrm{p}<0.0005)$. Developing cortex also had the lowest levels of $\mathrm{mBDNF}$, proBDNF and the lowest $\mathrm{mBDNF} /$ proBDNF ratio in comparison with other brain regions of 4-day old rat pups, as it was revealed by immunoblot (Fig. 2a; $\mathrm{mBDNF}-\mathrm{F}_{(3,21)}=12.79, \mathrm{p}<0.0001 ;$ proBDNF $\mathrm{F}_{(3,21)}=5.50, \quad \mathrm{p}<0.006 ; \quad \mathrm{mBDNF} / \mathrm{proBDNF}$ ratio $\left.\mathrm{F}_{(3,21)}=9.85, \mathrm{p}<0.0003\right)$. In addition, developing cortex was the only region, in which proBDNF was expressed as the main form of bdnf gene (Fig. 2a).

Across studied brain regions, the expression of 
proBDNF was not associated with the expression of active caspase-3 (Fig. 2c; $\mathrm{R}=-0.179$, n.s.). At the same time, the levels of mBDNF protein were negatively linked with the levels of active form of apoptotic protease throughout the immature brain (Fig. 2b; $\mathrm{R}=-0.662$, $\mathrm{p}<0.001$ ), resulting in the strong association between mBDNF to proBDNF ratio and the levels of active caspase-3 (Fig. 2d; $\mathrm{R}=-0.804, \mathrm{p}<0.001$ ). (a)

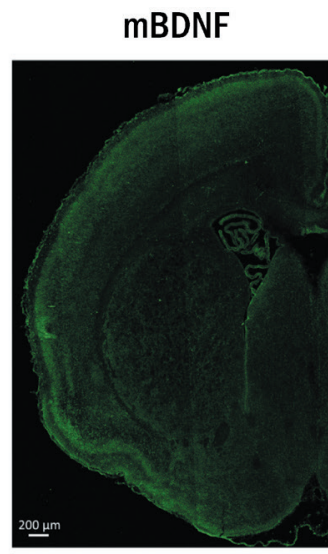

(b)

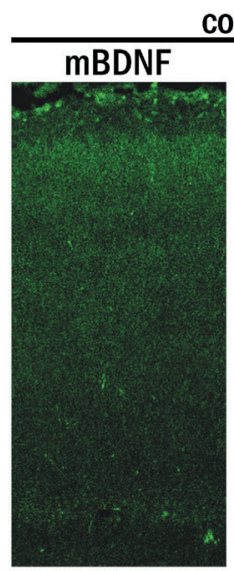

cortex

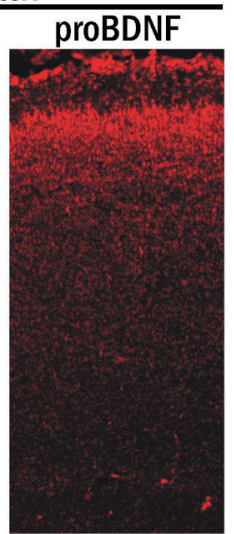

proBDNF

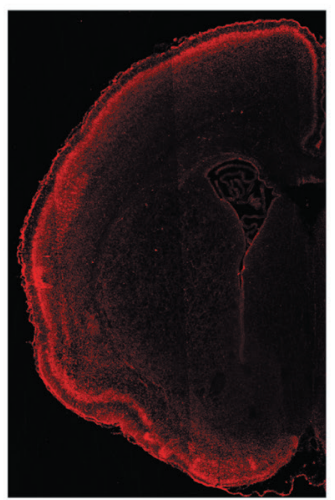

merge

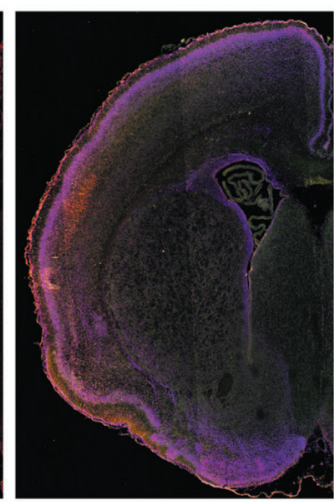

(c) Integral fluorescence

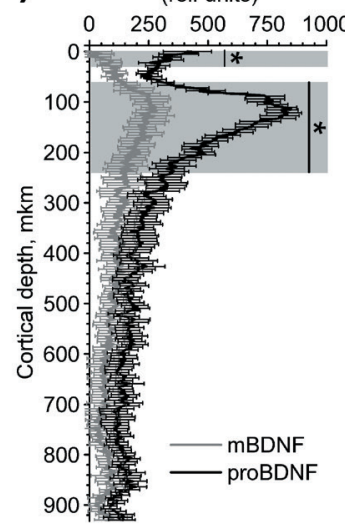

(d)
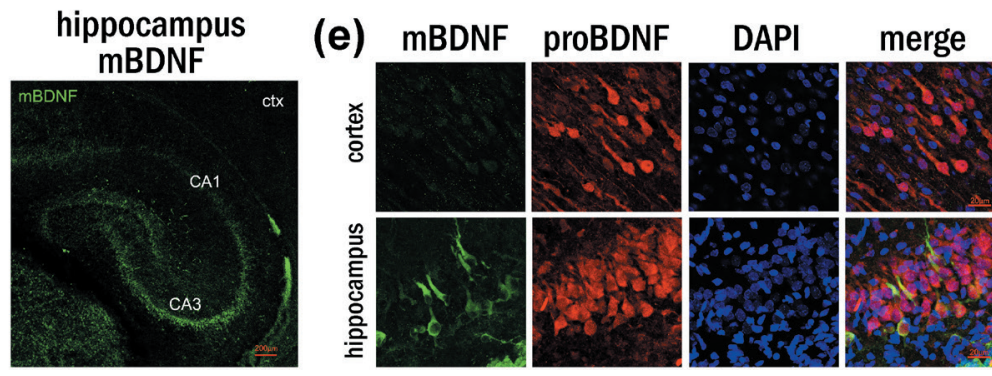

Fig. 3. Immunostaining for $m B D N F$ and proBDNF in the developing brain of P4 pups. (a) Immunostaining for mBDNF and proBDNF proteins in the whole brain frontal section, lambda -2 . Both proteins were mainly localized in upper layers of the cerebral cortex. Scale bar $=200 \mu \mathrm{m}$. (b) Immunostaining pattern of $\mathrm{mBDNF}$ and proBDNF in the developing cortex. (c) Integral quantification of fluorescence signal intensity for mBDNF and proBDNF protein levels. $*-p<0.05$ vs mBDNF levels at the same depth. Data are presented as the means \pm SEM. (d) Immunostaining pattern of mBDNF in the developing hippocampus and adjacent cortex. Scale bar $=200 \mu \mathrm{m}$. (e) High-magnification images of cells expressing mBDNF and proBDNF in the layer 2-3 of developing cortex and CA3 field of hippocampus. Scale bar $=20 \mu \mathrm{m}$.
Immunohistochemical detection of proBDNF and $m B D N F$

Cerebral cortex had the complex layered structure. To find out which of the layers contributes the most to the unique cortical mBDNF to proBDNF ratio, the immunohistochemical analysis of the radial distribution of BDNF forms was performed at this brain structure. In agreement with the immunoblot data, proBDNF was the main BDNF form detected in the cortex by immunohistochemistry, and its expression reached the highest levels in the layers 2-3 (Fig. 3a-c;
Cortical distribution $-\mathrm{F}_{(678,5424)}=5.68, \quad \mathrm{p}<0.0001$; Cortical distribution*BDNF form $-\mathrm{F}_{(678,5424)}=18.37$, $\mathrm{p}<0.0001$; BDNF form $\left.-\mathrm{F}_{(1,8)}=4.07, \mathrm{p}<0.079\right)$. Though, mBDNF staining was weaker through the cortical layers, nevertheless, its spatial distribution was positively correlated with the spatial distribution of proBDNF (Fig. 3b-c; $\mathrm{R}=0.857 ; \mathrm{p}<0.0001$ ). In contrast to the cortex, numerous mBDNF-positive cells were readily detected in the hippocampus - especially in the CA3 field of this brain region (Fig. 3d). 


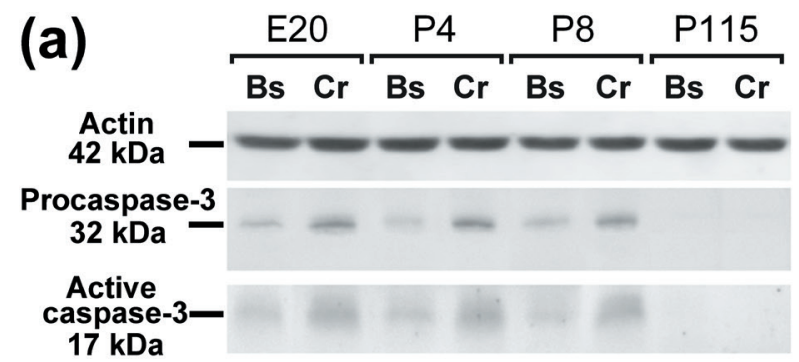

(b)

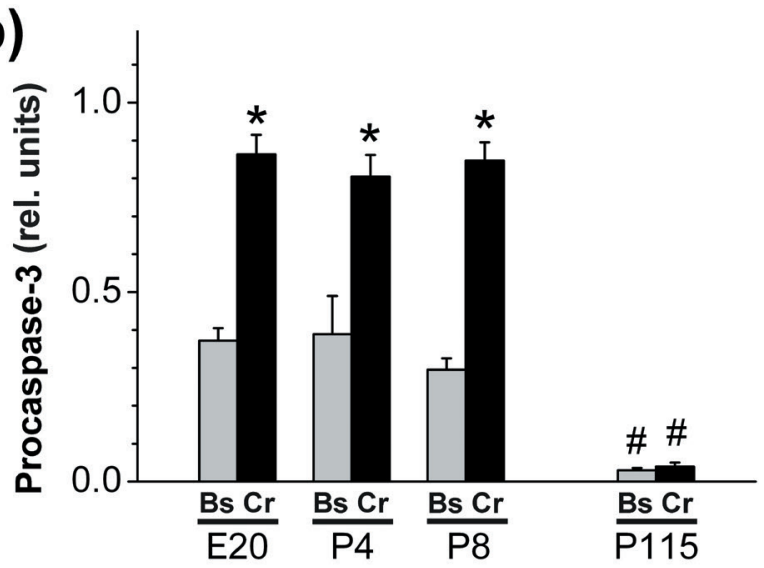

(c)

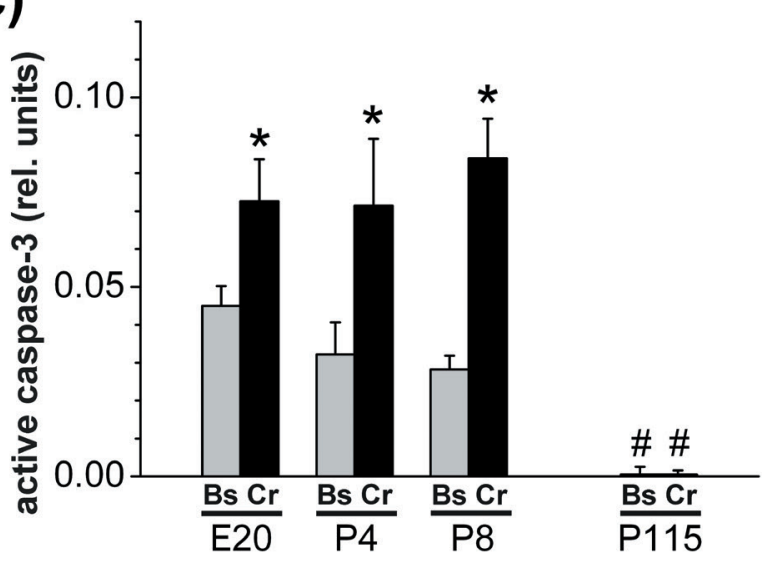

(d)

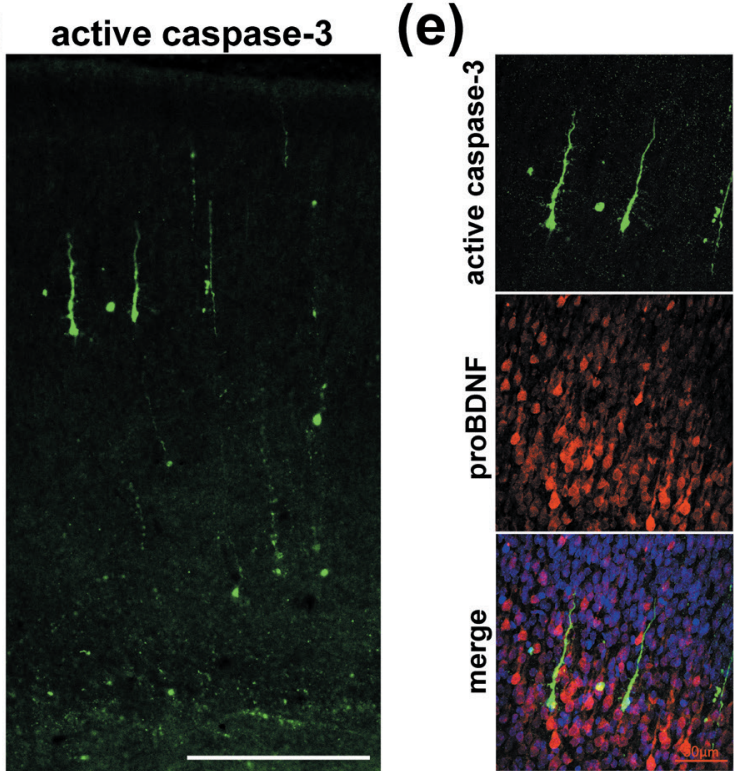

Fig. 4. Expression of caspase- 3 in the rat cortex and brainstem. (a) Immunoblot with procaspase-3 (32 kDa), active caspase-3 $(17 \mathrm{kDa})$ and actin $(42 \mathrm{kDa})$ bands. (b) Procaspase-3 protein levels. (c) Active caspase-3 protein levels. * $*^{-} p<0.05$ vs brainstem. \# - $p<0.05$ vs previous age. E20 - 20-day-old fetuses, P4 - 4-day-old pups, P8 - 8-day-old pups, P115 115-day-old adult rats. Bs - brainstem. Cr - cortex. Data are presented as the means \pm SEM. (d) Immunostaining pattern of active caspase- 3 in the developing cortex. Scale bar $=200 \mu \mathrm{m}$. (e) High-magnification images of cells expressing active caspase-3 and proBDNF in the layer 2-3 of developing cortex. Scale bar $=50 \mu \mathrm{m}$.

High magnification of the cortical layer 2-3 revealed abundant proBDNF expression in multiple cell bodies and axons of neurons located in this region (Fig. 3e). Only a very faint mBDNF signal was detected in the neurons of the cortical layer 2-3 (Fig. 3e). Similar to the cortex, multiple neurons were also proBDNFpositive in the CA3 area of hippocampus (Fig. 3e). However, unlike in the cortex, proBDNF staining in the hippocampal neurons was generally localized in the cell bodies, while processes were mostly proBDNF-negative. Not all proBDNF-positive cells in the CA3 region were mBDNF-positive too. Moreover, in contrast to proBDNF, mature neurotrophin was readily detectable either in the cell bodies and axons of CA3 cells (Fig. 3e).

Correlations between proBDNF and active caspase-3 levels in the cortex and brainstem

Both BDNF forms could have effects on the expression of active caspase-3 (Koshimizu et al. 2010). The unique low ratio of $\mathrm{mBDNF} /$ proBDNF in the developing cortex suggests the existence of a specific interrelation between proBDNF and active caspase-3 levels in this brain structure. To analyze such possibility, the local intraregional comparison of proBDNF and active caspase- 3 expression in the developing cortex and brainstem was done. Both forms of caspase-3 were detected in the developing brain on E20, P4 and P8 days, with the higher levels found in the cortex than in the brainstem (Fig. 4). In the cortex, sparse active caspase-3positive cells were localized in the layers 2-3 and layer 5 (Fig. 4d-e). The significant intraregional positive correlation between the expression of proneurotrophin and active caspase- 3 was found only in the cortex (Fig. 5a; $\mathrm{R}=0.577, \mathrm{p}<0.020$ ), while there were no such correlation in the brainstem (Fig. $5 \mathrm{~b} ; \mathrm{R}=-0.232$, n.s. for proBDNF). Levels of procaspase- 3 and active caspase- 3 in the cortex and brainstem of adult animals were very low in comparison to perinatal samples (Fig. 4). 
(a)
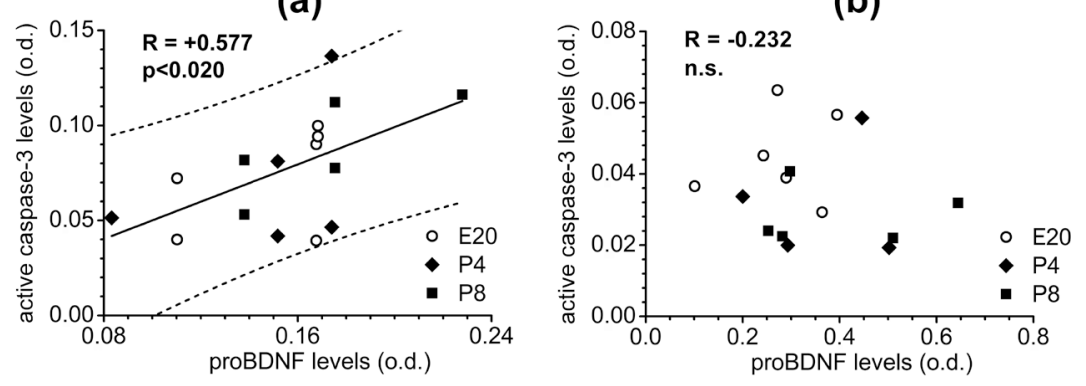

Fig. 5. Interrelations of active caspase-3 expression with the levels of proBDNF in the developing cortex (a) and brainstem (b). E20 - 20-day-old fetuses, P4 - 4-day-old pups, P8 - 8-day-old pups, P115 - 115-dayold adult rats. Solid lines mark the regressions; short dot lines mark $90 \%$ prediction intervals for regressions.

\section{Discussion}

Here we provide the first evidence that immature cortex was the only brain region, in which proBDNF was the major product of $b d n f$ gene, and its expression positively correlated with the levels of active caspase-3.

mBDNF levels vary greatly across brain regions in the adult brain (Gray et al. 2013). The cortex, brainstem, hippocampus and cerebellum of our pups were also characterized by the high variance in the expression of mature neurotrophin. However, the levels of mBDNF were negatively correlated with the levels of active caspase- 3 in these brain regions. The lowest expression of mature neurotrophin as well as the highest expression of apoptotic protease was found in the immature cortex. The negative correlation between the levels of mBDNF and apoptotic protease across neonatal brain regions was in line with the neurotrophic theory, suggesting antiapoptotic effects of mature neurotrophins in the developing brain (Dekkers et al. 2013). Its looks like proBDNF did not exert any proapoptotic function in the developing brain in the presence of substantial quantities of mature neurotrophin, as it could be seen from the distribution of active caspase- 3 throughout CNS regions. This notion was supported by data of Koshimizu et al. (2010), who observed the suppression of proBDNFmediated cell death by mBDNF.

Perinatal cortex was the only brain structure, in which proBDNF was the predominant form of BDNF. High amounts of proneurotrophin in this region found for the first time in our work were quite unexpected due to the fact that the levels of mature form of BDNF were low during cortical development, as it was shown in the present and other studies (Katoh-Semba et al. 1997, Huang et al. 1999, Rossi et al. 1999, Das et al. 2001, Kim et al. 2007, Bracken and Turrigiano 2009, Lasley and Gilbert 2011). The disproportion between the cortical levels of proBDNF and mBDNF may result from either low conversion rates of proneurotrophin into the mature form and/or rapid clearance of mBDNF, as it was shown for the adult hippocampus (Patz and Wahle 2004).

The importance of mBDNF to proBDNF ratio and its rapid change has been shown earlier for the processes occurred in the adult CNS, such as synapse development and plasticity, axon guidance and memory formation (Koshimizu et al. 2009, Marler et al. 2010, Je et al. 2012). However, in contrast to adult brain, the predominance of proBDNF in the developing cortex was not a short kinetic phenomenon, and the ratio of $\mathrm{mBDNF}$ to proBDNF was relatively constant during the whole perinatal period. The absence of substantial changes in the ratio of BDNF forms may reflect the importance of these molecules as regulators of cell survival during brain development, because the functions of mature neurotrophin and its precursor in the immature brain may be different from their roles in the adult CNS (Patz and Wahle 2004).

Immunohistochemical analysis demonstrated that both proBDNF and mBDNF were localized mainly in the superficial cortical layers 2-3, the development of which continued during early postnatal life (Kohwi and Doe 2013). proBDNF expression was widespread not only in the neurons of cortical layers 2-3, but also in the hippocampus. The similar pattern of proBDNF staining was detected in the hippocampus of neonatal rats by Langlois et al. (2013) and mice by Yang et al. (2009). However, multiple proBDNF-positive staining of axons was detected in the cortical neurons only, while hippocampal cell processes were mostly proBDNFnegative.

When secreted from cells, both forms of BDNF could affect the levels of active caspase-3 (Dekkers et al. 2013). Indeed, active caspase-3-positive cells were found in the developing cortex of P4 animals, particularly in the layer 2-3, in which most proBDNF-positive cells were located (Fig. 5d-e). However, neither immunohistochemistry, no immunoblot analysis was able to estimate the ratio of extracellular to intracellular mBDNF or proBDNF in the cortex. Moreover, several groups do not find any convenient evidences for the release or 
abundant presence of extracellular proBDNF in the brain (for example Matsumoto et al. 2008). Nevertheless, if proBDNF had any functional role in the cortex, then the expression of active caspase- 3 should follow proneurotrophin levels. We found positive correlation between proBDNF and active caspase-3 expression in the developing cortex. This correlation suggests that at least some amount of proBDNF found in the forebrain might be excreted and had its own function in the cortical development. Nevertheless, other, more sophisticated methods should be used to evaluate the amount of extracellular proBDNF during cortical development.

We also found that proBDNF levels in the cortex and brainstem of our adult animals were much higher in comparison to the developing brain. Our results are in line with the work of Perovic et al. (2013) who reported that both proBDNF and mBDNF were abundantly present in the cerebral cortex of adult rats until the senescence. While proBDNF expressed in the adult CNS might be involved in the synapse plasticity and memory formation (Patz and Wahle 2004), it is unlikely, that high cortical and brainstem levels of this proneurotrophin could be linked to cell death due to the absence of both procaspase- 3 and active caspase- 3 in these brain regions.

It is possible that, if secreted, proBDNF might act as an initial death signal inducing basal rates of physiological cell death in the developing cortex. However, it is obvious that such proapoptotic action of proBDNF would be hard to discover in the presence of different antiapoptotic factors. It also should be noted that proBDNF could be converted to mBDNF by endopeptidases extracellularly (Lessmann and Brigadski 2009). However, the functional role of $\mathrm{mBDNF}$ in the developing cortex remains largely unclear (Linnarsson et al. 2000, Southwell et al. 2012).

In summary, our data provide the first evidence that proBDNF is the predominant form of BDNF expressed in the developing rat cortex, and its expression in this brain structure correlates with the levels of active caspase-3 during perinatal development. These findings support the suggestion that proBDNF alone may play an important role in regulation of naturally occurring cell death in the developing cortex. In contrast to the cortex, in other brain regions studied the levels of mBDNF and proBDNF were comparable, and mBDNF levels were negatively correlated with the levels of active caspase-3. As a result, the strong interregional negative correlation between $\mathrm{mBDNF}$ to proBDNF ratio and active caspase-3 levels exists throughout the immature brain, and high levels of mature neurotrophin may shadow the significance of BDNF precursor as the cell death inductor in the developing brainstem, hippocampus and cerebellum.

\section{Conflict of Interest}

There is no conflict of interest.

\section{Acknowledgements}

This study was supported by RFBR grants 14-0400219_a, 15-04-05602_a, 14-04-31447-mol_a, MES RF grant MK-1207.2014.4 and FASO VI.53.2.2. All microscopy imaging was done at the ICG SB RAS Microscopy Core.

\section{Abbreviations}

BDNF - brain derived neurotrophic factor, proBDNF BDNF precursor, mBDNF - mature BDNF.

\section{References}

BRACKEN BK, TURRIGIANO GG: Experience-dependent regulation of TrkB isoforms in rodent visual cortex. Dev Neurobiol 69: 267-278, 2009.

DAS KP, CHAO SL, WHITE LD, HAINES WT, HARRY GJ, TILSON HA, BARONE S JR: Differential patterns of nerve growth factor, brain-derived neurotrophic factor and neurotrophin-3 mRNA and protein levels in developing regions of rat brain. Neuroscience 103: 739-761, 2001.

DEKKERS MP, NIKOLETOPOULOU V, BARDE YA: Cell biology in neuroscience: Death of developing neurons: New insights and implications for connectivity. J Cell Biol 203: 385-393, 2013.

EGASHIRA Y, TANAKA T, SONI P, SAKURAGI S, TOMINAGA-YOSHINO K, OGURA A: Involvement of the p75(NTR) signaling pathway in persistent synaptic suppression coupled with synapse elimination following repeated long-term depression induction. J Neurosci Res 88: 3433-3446, 2010.

FAN YJ, WU LL, LI HY, WANG YJ, ZHOU XF: Differential effects of pro-BDNF on sensory neurons after sciatic nerve transection in neonatal rats. Eur J Neurosci 27: 2380-2390, 2008. 
FAYARD B, LOEFFLER S, WEIS J, VOGELIN E, KRUTTGEN A: The secreted brain-derived neurotrophic factor precursor pro-BDNF binds to TrkB and p75NTR but not to TrkA or TrkC. J Neurosci Res 80: 18-28, 2005.

GHOSH A, CARNAHAN J, GREENBERG ME: Requirement for BDNF in activity-dependent survival of cortical neurons. Science 263: 1618-1623, 1994.

GIEHL KM, RÖHRIG S, BONATZ H, GUTJAHR M, LEINER B, BARTKE I, YAN Q, REICHARDT LF, BACKUS C, WELCHER AA, DETHLEFFSEN K, MESTRES P, MEYER M: Endogenous brain-derived neurotrophic factor and neurotrophin-3 antagonistically regulate survival of axotomized corticospinal neurons in vivo. J Neurosci 21: 3492-3502, 2001.

GRAY JD, MILNER TA, MCEWEN BS: Dynamic plasticity: the role of glucocorticoids, brain-derived neurotrophic factor and other trophic factors. Neuroscience 239: 214-227, 2013.

HAN BH, HOLTZMAN DM: BDNF protects the neonatal brain from hypoxic-ischemic injury in vivo via the ERK pathway. J Neurosci 20: 5775-5781, 2000.

HAN BH, D'COSTA A, BACK SA, PARSADANIAN M, PATEL S, SHAH AR, GIDDAY JM, SRINIVASAN A, DESHMUKH M, HOLTZMAN DM: BDNF blocks caspase-3 activation in neonatal hypoxia-ischemia. Neurobiol Dis 7: 38-53, 2000.

HEAD BP, PATEL HH, NIESMAN IR, DRUMMOND JC, ROTH DM, PATEL PM: Inhibition of p75 neurotrophin receptor attenuates isoflurane-mediated neuronal apoptosis in the neonatal central nervous system. Anesthesiology 110: 813-825, 2009.

HUANG ZJ, KIRKWOOD A, PIZZORUSSO T, PORCIATTI V, MORALES B, BEAR MF, MAFFEI L, TONEGAWA S: BDNF regulates the maturation of inhibition and the critical period of plasticity in mouse visual cortex. Cell 98: 739-755, 1999.

HUNG PL, HUANG CC, HUANG HM, TU DG, CHANG YC: Thyroxin treatment protects against white matter injury in the immature brain via brain-derived neurotrophic factor. Stroke 44: 2275-2283, 2013.

JE HS, YANG F, JI Y, NAGAPPAN G, HEMPSTEAD BL, LU B: Role of pro-brain-derived neurotrophic factor (proBDNF) to mature BDNF conversion in activity-dependent competition at developing neuromuscular synapses. Proc Natl Acad Sci USA 109: 15924-15929, 2012.

KATOH-SEMBA R, TAKEUCHI IK, SEMBA R, KATO K: Distribution of brain-derived neurotrophic factor in rats and its changes with development in the brain. J Neurochem 69: 34-42, 1997.

KENCHAPPA RS, ZAMPIERI N, CHAO MV, BARKER PA, TENG HK, HEMPSTEAD BL, CARTER BD: Liganddependent cleavage of the P75 neurotrophin receptor is necessary for NRIF nuclear translocation and apoptosis in sympathetic neurons. Neuron 50: 219-232, 2006.

KIM JK, JEON SM, LEE KM, PARK ES, CHO HJ: Expression of brain-derived neurotrophic factor in the rat forebrain and upper brain stem during postnatal development: an immunohistochemical study. Neuroscience 146: 1128$1136,2007$.

KOHWI M, DOE CQ: Temporal fate specification and neural progenitor competence during development. Nat Rev Neurosci 14: 823-838, 2013.

KOKAIA Z, NAWA H, UCHINO H, ELMÉR E, KOKAIA M, CARNAHAN J, SMITH ML, SIESJÖ BK, LINDVALL $\mathrm{O}$ : Regional brain-derived neurotrophic factor mRNA and protein levels following transient forebrain ischemia in the rat. Brain Res Mol Brain Res 38: 139-144, 1996.

KOSHIMIZU H, KIYOSUE K, HARA T, HAZAMA S, SUZUKI S, UEGAKI K, NAGAPPAN G, ZAITSEV E, HIROKAWA T, TATSU Y, OGURA A, LU B, KOJIMA M: Multiple functions of precursor BDNF to CNS neurons: negative regulation of neurite growth, spine formation and cell survival. Mol Brain 2: 27, 2009.

KOSHIMIZU H, HAZAMA S, HARA T, OGURA A, KOJIMA M: Distinct signaling pathways of precursor BDNF and mature BDNF in cultured cerebellar granule neurons. Neurosci Lett 473: 229-232, 2010.

LANGLOIS A, DIABIRA D, FERRAND N, PORCHER C, GAIARSA JL: NMDA-dependent switch of proBDNF actions on developing GABAergic synapses. Cereb Cortex 23: 1085-1096, 2013.

LANSHAKOV DA, BULYGINA VV, ROMANOVA IV, DYGALO NN: Immunohistochemical analysis of active caspase-3 expression in structures of neonatal brain. Bull Exp Biol Med 147: 635-638, 2009.

LASLEY SM, GILBERT ME: Developmental thyroid hormone insufficiency reduces expression of brain-derived neurotrophic factor (BDNF) in adults but not in neonates. Neurotoxicol Teratol 33: 464-472, 2011. 
LESSMANN V, BRIGADSKI T: Mechanisms, locations, and kinetics of synaptic BDNF secretion: an update. Neurosci Res 65: 11-22, 2009.

LINNARSSON S, WILLSON CA, ERNFORS P: Cell death in regenerating populations of neurons in BDNF mutant mice. Brain Res Mol Brain Res 75: 61-69, 2000.

MADEDDU F, NASKA S, BOZZI Y: BDNF down-regulates the caspase-3 pathway in injured geniculo-cortical neurones. Neuroreport 15: 2045-2049, 2004.

MARLER KJ, POOPALASUNDARAM S, BROOM ER, WENTZEL C, DRESCHER U: Pro-neurotrophins secreted from retinal ganglion cell axons are necessary for ephrinA-p75NTR-mediated axon guidance. Neural Dev 5: $30,2010$.

MATSUMOTO T, RAUSKOLB S, POLACK M, KLOSE J, KOLBECK R, KORTE M, BARDE YA: Biosynthesis and processing of endogenous BDNF: CNS neurons store and secrete BDNF, not pro-BDNF. Nat Neurosci 11: 131-133, 2008.

MEN'SHANOV PN, MUZYKA VV, DYGALO NN: Coordinated expression of pro- and antiapoptotic proteins in the hippocampus of neonatal rats. Neurochem $J$ 5: 20-23, 2011.

MENSHANOV PN, BANNOVA AV, DYGALO NN: Region-specific interrelations between apoptotic proteins expression and DNA fragmentation in the neonatal rat brain. Neurochem Res 31: 869-875, 2006.

MOONEY SM, MILLER MW: Expression of bcl-2, bax, and caspase-3 in the brain of the developing rat. Brain Res Dev Brain Res 123: 103-117, 2000.

MUZYKA VV, MEN'SHANOV PN, BANNOVA AV, DYGALO NN: The interrelationship between BDNF and its precursor and the level of active caspase-3 in the brain regions of neonatal rats. Neurochem $J$ 6: 260-264, 2012.

NAGAPPAN G, ZAITSEV E, SENATOROV VV JR, YANG J, HEMPSTEAD BL, LU B: Control of extracellular cleavage of ProBDNF by high frequency neuronal activity. Proc Natl Acad Sci USA 106: 1267-1272, 2009.

NAKAJIMA H, UCHIDA K, YAYAMA T, KOBAYASHI S, GUERRERO AR, FURUKAWA S, BABA H: Targeted retrograde gene delivery of brain-derived neurotrophic factor suppresses apoptosis of neurons and oligodendroglia after spinal cord injury in rats. Spine (Phila Pa 1976) 35: 497-504, 2010.

PATZ S, WAHLE P: Neurotrophins induce short-term and long-term changes of cortical neurotrophin expression. Eur $J$ Neurosci 20: 701-708, 2004.

PEROVIC M, TESIC V, MLADENOVIC DJORDJEVIC A, SMILJANIC K, LONCAREVIC-VASILJKOVIC N, RUZDIJIC S, KANAZIR S: BDNF transcripts, proBDNF and proNGF, in the cortex and hippocampus throughout the life span of the rat. Age (Dordr) 35: 2057-2070, 2013.

ROSSI FM, BOZZI Y, PIZZORUSSO T, MAFFEI L: Monocular deprivation decreases brain-derived neurotrophic factor immunoreactivity in the rat visual cortex. Neuroscience 90: 363-368, 1999.

SOUTHWELL DG, PAREDES MF, GALVAO RP, JONES DL, FROEMKE RC, SEBE JY, ALFARO-CERVELLO C, TANG Y, GARCIA-VERDUGO JM, RUBENSTEIN JL, BARABAN SC, ALVAREZ-BUYLLA A: Intrinsically determined cell death of developing cortical interneurons. Nature 491: 109-113, 2012.

TANG S, MACHAALANI R, WATERS KA: Brain-derived neurotrophic factor (BDNF) and TrkB in the piglet brainstem after postnatal nicotine and intermittent hypercapnic hypoxia. Brain Res 1232: 195-205, 2008.

TANG S, MACHAALANI R, WATERS KA: Immunolocalization of pro- and mature-brain derived neurotrophic factor (BDNF) and receptor TrkB in the human brainstem and hippocampus. Brain Res 1354: 1-14, 2010.

TENG HK, TENG KK, LEE R, WRIGHT S, TEVAR S, ALMEIDA RD, KERMANI P, TORKIN R, CHEN ZY, LEE FS, KRAEMER RT, NYKJAER A, HEMPSTEAD BL: ProBDNF induces neuronal apoptosis via activation of a receptor complex of p75NTR and sortilin. $J$ Neurosci 25: 5455-5463, 2005.

WANG H, WU LL, SONG XY, LUO XG, ZHONG JH, RUSH RA, ZHOU XF: Axonal transport of BDNF precursor in primary sensory neurons. Eur J Neurosci 24: 2444-2452, 2006.

XU ZQ, SUN Y, LI HY, LIM Y, ZHONG JH, ZHOU XF: Endogenous proBDNF is a negative regulator of migration of cerebellar granule cells in neonatal mice. Eur J Neurosci 33: 1376-1384, 2011.

YANG J, SIAO CJ, NAGAPPAN G, MARINIC T, JING D, MCGRATH K, CHEN ZY, MARK W, TESSAROLLO L, LEE FS, LU B, HEMPSTEAD BL: Neuronal release of proBDNF. Nat Neurosci 12: 113-115, 2009. 